\title{
Erratum: Quantum tasks in holography
}

\author{
Alex May \\ Department of Physics and Astronomy, The University of British Columbia, \\ Vancouver, BC, Canada \\ E-mail: may@phas.ubc.ca
}

ERRATUM TO: JHEP10(2019)233

ARXIV EPRINT: 1902.06845

As one of the results in this article, I stated a connection between bulk causal structure and boundary entanglement in the AdS/CFT correspondence. This statement appears in the introduction and section 3.3. This connection followed by relating results on the necessity of entanglement to perform certain distributed computations non-locally. At a technical level bringing these necessity of entanglement results in contact with AdS/CFT is challenging, and in fact an error occurred in the published article.

The article defines two in-regions $R_{i}$, defined in equation 3.6. These two regions are spacelike separated and can be extended to a complete Cauchy slice of the boundary CFT. Call such a slice $\Sigma$. Note that $\Sigma$ contains two spacetime regions $X_{1}, X_{2}$ that sit between $R_{1}, R_{2}$. Theorem 7 of the article followed by arguing that the boundary CFT needed a mutual information of order $1 / G$ between $R_{1}$ and $R_{2}$ for the boundary CFT to reproduce processes that can occur in the bulk. In doing so, we had to argue that the degrees of freedom $G_{1}$ and $G_{2}$ sitting in the spacetime region $X_{1}$ and $X_{2}$ did not assist in realizing the bulk process in the boundary. In part, we handled this by exploiting the structure of quantum Markov states.

Unfortunately definition 9 and theorem 11 concerning Markov states are not true, and amount to an error on the part of the author. Consequently, theorems 5, 6 and 7 should be placed at the level of conjectures. Because the regions $X_{i}$ are spacelike separated from the input points $c_{i}$, it is plausible that they are in fact not involved in the boundary's realizing of the bulk process. If we were to assume this the conjectures would be proven. Further, we provided checks in a specific simple example in appendix $\mathrm{C}$ of theorem 7 . These "checks" should now be viewed as evidence for the conjecture. 
While this error is certainly regretable, the conjectures are actually true - in a more recent article [1] the authors proved theorem 7 using techniques from classical general relativity. Further, once theorem 7 is established something very close to theorem 6 follows: a connected entanglement wedge implies order $1 / G$ mutual information, so that indeed the mutual information between the in-regions is large.

The remaining sections of the article remain unchanged. A version incorporating the above corrections can be found on the arXiv [2]. A definitive treatment of the content of section 3.3 can be found in the more recent related article [1].

Open Access. This article is distributed under the terms of the Creative Commons Attribution License (CC-BY 4.0), which permits any use, distribution and reproduction in any medium, provided the original author(s) and source are credited.

\section{References}

[1] A. May, G. Penington and J. Sorce, Holographic scattering requires a connected entanglement wedge, arXiv: 1912.05649 [INSPIRE].

[2] A. May, Quantum tasks in holography, JHEP 10 (2019) 233 [arXiv:1902.06845] [INSPIRE]. 